\title{
MORTALITY IN SWEDEN RELATED TO OCCUPATIONAL
} EXPOSURES

Bengt Jarvholm, ${ }^{1}$ Christina Reuterwall, ${ }^{1}$ Jennie Bystedt ${ }^{2}{ }^{1}$ Umeå University, Umeå, Sweden; ${ }^{2}$ Sundsvalls Hospital, Sundsvall, Sweden

\subsection{6/oemed-2011-100382.188}

Objectives The objective is to estimate to proportion of mortality in cancer, cardiovascular and respiratory diseases that can be attributed to occupational exposure.

Methods Sites of cancer that in IARC categories I and Ila were considered along with myocardial infarction, asthma, chronic obstructive lung disease, pneumoconioses and hypersensitivity pneumonitis. Most analyses were restricted to ages between 25 and 74 years of age. We used conservative estimates, when possible from national case-reference studies.

Results In total we estimated that there were around 800 deaths in 2007 attributable to occupational exposures in this age group. The majority were myocardial infarction $(\mathrm{N}=463)$ attributable to job-strain ( $\mathrm{N}=193)$, shift work $(\mathrm{N}=89)$ exhausts from motors and burning ( $\mathrm{N}=152)$ and environmental tobacco smoke $(\mathrm{N}=29)$. In total 270 deaths of cancer were attributed to occupational factors, approximately half of them in lung cancer $(\mathrm{N}=134)$. Ninety percent of mesothelioma deaths were estimated to be attributed to occupational factors meaning a total 56 cases (54 men and 2 women). There were 2 deaths in pneumoconiosis and no death in hypersensitivity pneumonitis in this age group. There were 4 deaths in asthma and 92 deaths in chronic obstructive lung disease attributed to occupational factors.

Conclusions Cardiovascular diseases contribute to a considerable proportion of the mortality related to occupational factors in the working population. Both psychosocial factors and exhaustive gases from engines and combustion seem important. Estimations of attributable deaths may be a useful tool in setting priorities in preventive strategies. 